\title{
Vibration Isolation of Biomedical Engineering Facilities with Periodic Materials
}

\author{
YL Mo* \\ Structural Engineering, University of Houston, USA \\ Submission: February 03, 2018; Published: March 26, 2018 \\ *Corresponding author: YL Mo, Structural Engineering, University of Houston, USA, Email: yilungmo@central.uh.edu
}

\section{Conventional Vibration Isolation Systems}

Vibrations have always been a serious threat to biomedical engineering facilities. To prevent the vibration effects on the facilities, many kinds of vibration isolation systems have been proposed and implemented in practice. The basic concept of the conventional vibration isolation, such as the Lead-Rubber Isolation System (LRB), is to introduce a soft layer between the biomedical engineering facility and foundation, so that the natural period of the biomedical engineering facility in the horizontal direction can be lengthened. The input vibration force that is transmitted from the foundation to the biomedical engineering facility can be reduced. However, such systems have major disadvantages. The large relative displacement between the non-isolated foundation and the isolated biomedical engineering facility that occurs during a vibration event demands special handling. These nonstructural elements need to be designed to accommodate these large displacements. Moreover, the conventional vibration isolation systems are ineffective in protecting the biomedical engineering facilities in the vertical direction of vibration. Therefore, a new vibration isolation system that eliminates such disadvantages would be very attractive.

\section{Innovative Concept of Periodic Materials}

A novel idea on passive vibration control created by adopting the concept of phononic crystal in solid-state physics, also called a periodic material-based vibration isolation system or periodic foundation, is analytically and experimentally investigated. Periodic foundation refers to a foundation made of periodic materials. Periodic materials, or metamaterials, can be constructed by arranging the unit cell in a repeated manner. Different from the conventional vibration isolation, the periodic material doesn't lengthen the natural period to reduce the vibration load but simply doesn't let the vibration propagate through the periodic material when the frequency of the incoming vibration waves is within the certain range of frequency (frequency band gaps). Figure 1 shows the characteristics of wave propagation in theoretically infinite periodic materials. The wave shown in Figure 1(a) cannot propagate into the periodic material when the frequency of the wave falls within the range of the frequency band gap of the material. In contrast, the wave shown in Figure 1(b) can propagate into, and through, the periodic material, because the frequency of the wave is outside the range of the material's frequency band gap [1].

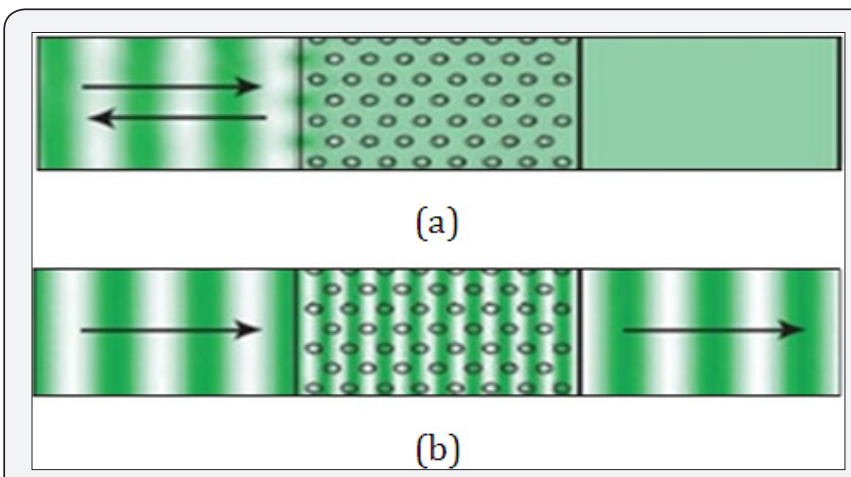

Figure 1: (a) Reflection of a wave possessing a frequency falling within the frequency band gap of the periodic material.

(b) Wave propagation of a wave possessing a frequency outside of the frequency band gap of the periodic material 1

Theoretically, a drastic reduction of the wave between the input and output is expected when the incoming waves have frequencies within the frequency band gaps of the periodic material. A frequency band gap is the property that periodic materials possess. The desired frequency band gaps can be obtained by the properties of composed materials, such as Young's modulus, Poisson ratio, thickness, etc. On top of their unique isolation mechanism, periodic foundations can sustain gravity loads of a biomedical engineering facility as well.

The research on periodic materials, also known as phononic crystals in the branch of solid state physics, has shown that an infinite series of lattice layers has the ability to manipulate certain elastic waves traveling through its medium. According to the number of directions where the unit cell is repeated, periodic materials or phononic crystals can be classified as onedimensional (1D), two-dimensional (2D) and three-dimensional (3D) phononic crystals, as shown in Figure 2 [2-4]. 


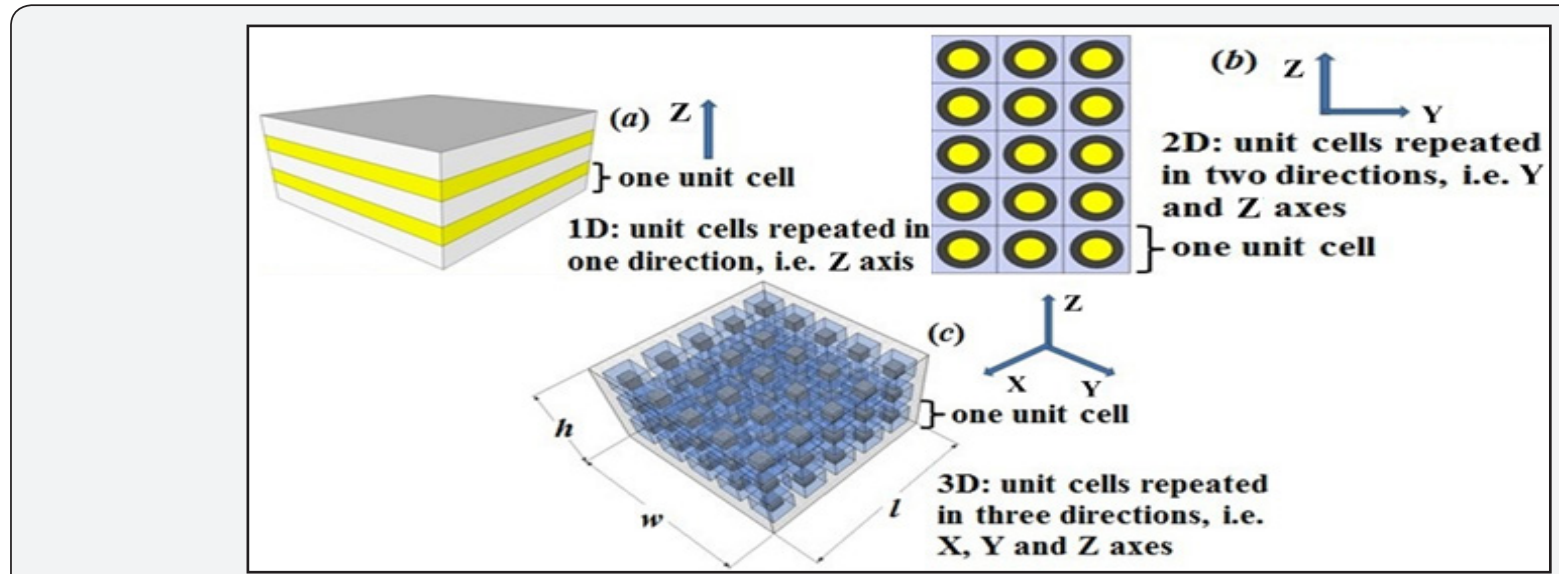

Figure 2 : Classification of periodic material (a) 1D (b) 2D (c) 3D

\section{Feasibility Study}

The University of Houston (UH) has conducted several analytical and experimental studies of periodic foundations. Figure 3 shows the experimental tests of 1D, 2D and 3D periodic foundations. The tests are to validate the fundamental theory of frequency band gaps in each of the periodic foundations. The simple structure supported by each of the 1D, 2D and 3D periodic foundations is marked as specimens B, D and F [2-4]. Identical structures without periodic foundations, marked as specimens A, C and E, are tested simultaneously with their counterparts. The test results presented in Figure 4 show that the facilities isolated with periodic foundations have a much lesser vibration response compared to non-isolated facilities.

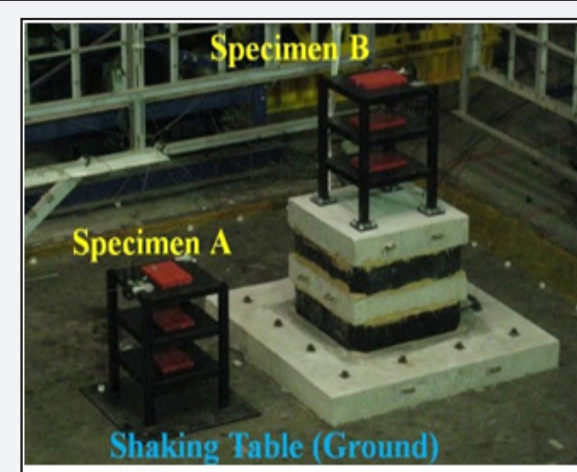

(a)

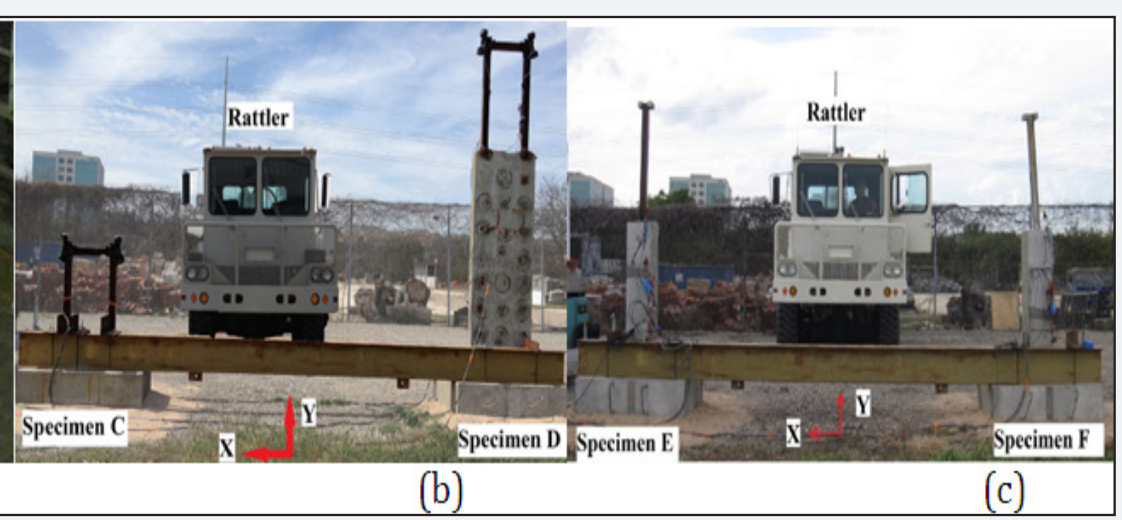

Figure 3 : Experimental test setup: (a) 1D periodic foundation at $\mathrm{NCREE}^{2}$; (b) 2D periodic foundation at $\mathrm{UT}^{3}$; and (c) 3D periodic foundation at UT.

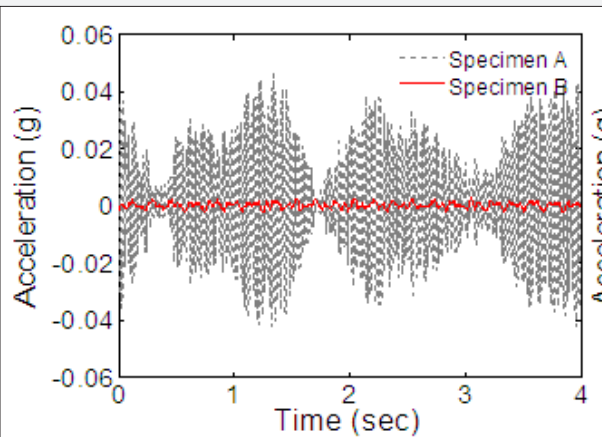

(a)

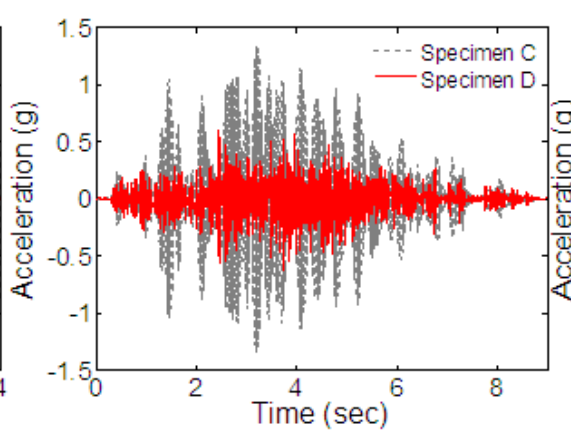

(b)

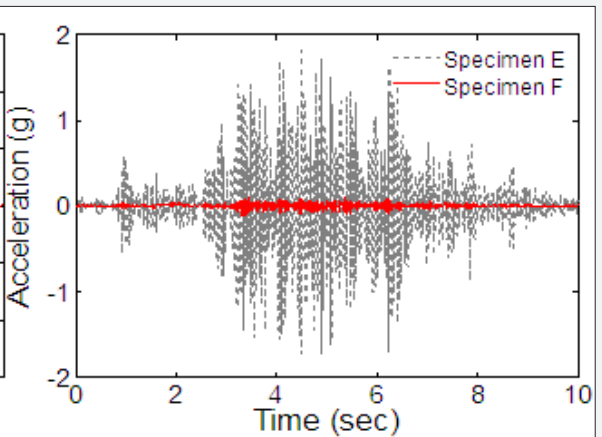

(c)

Figure 4 : Test results of (a) 1D periodic foundation (b) 2D periodic foundation and (c) 3D periodic foundation 


\section{Applications to Biomedical Engineering Facilities}

Following the test validations, UH proceeded to apply periodic materials to real facilities. In the subsequent tests, the UH team designed a four-layer 1D periodic foundation supporting a facility, shown in Figure 5. Shake table test results using Gilroy vibration ground motion show that the designed periodic materials can drastically reduce the vibration input by $90 \%$, Figure $6(\mathrm{a})$. The transfer function of the vibration records, shown in Figure 6(b), denote that the response of the top of the periodic material-based foundation and the roof were significantly diminished in the frequency range of $6.5-50 \mathrm{~Hz}$. This region matched with the designed frequency band gaps (yellow shaded areas), proving that the frequency band gaps theory works. The shake table tests demonstrate that periodic materials can be applied to the real biomedical engineering facilities.

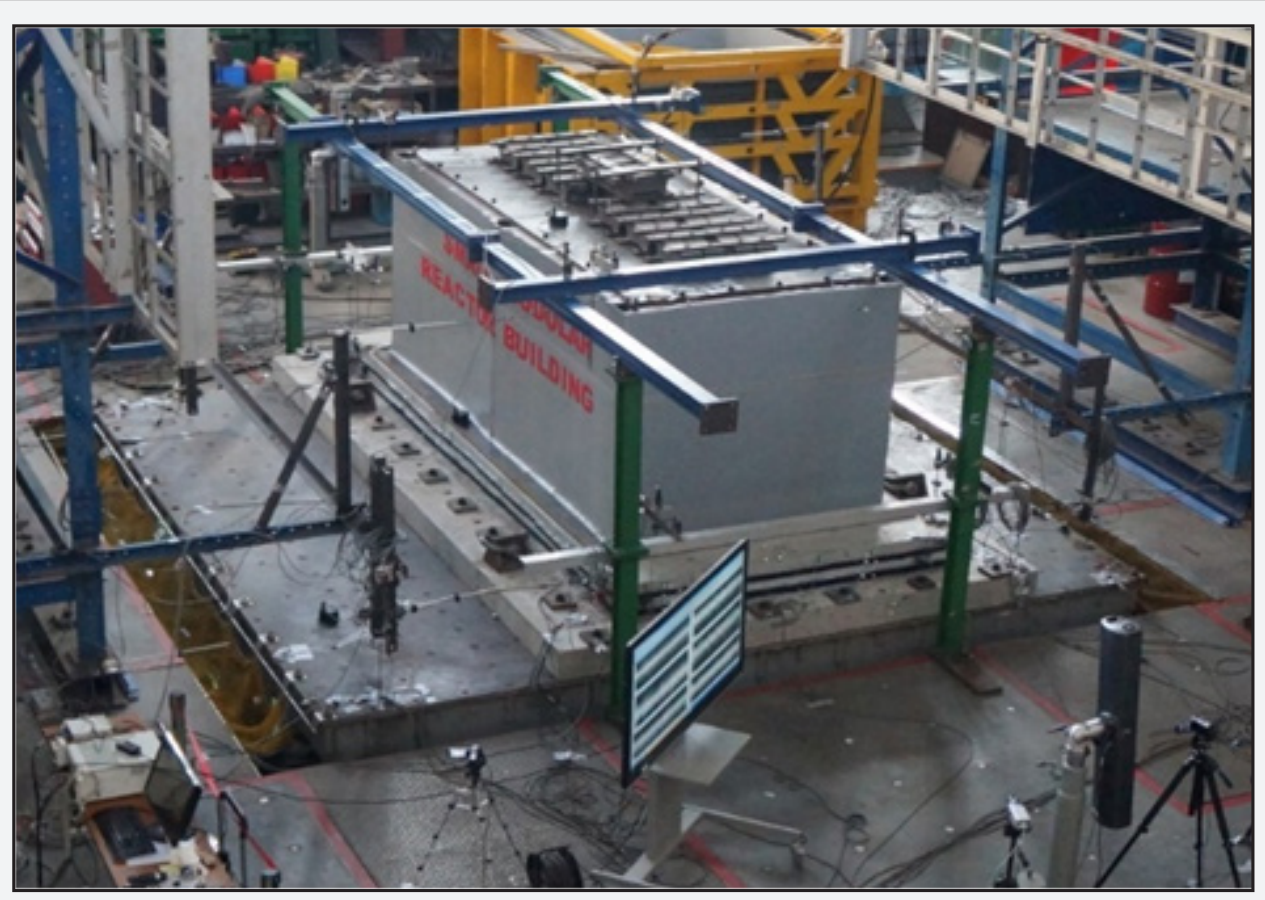

Figure 5: Facility supported by 1D periodic foundation.

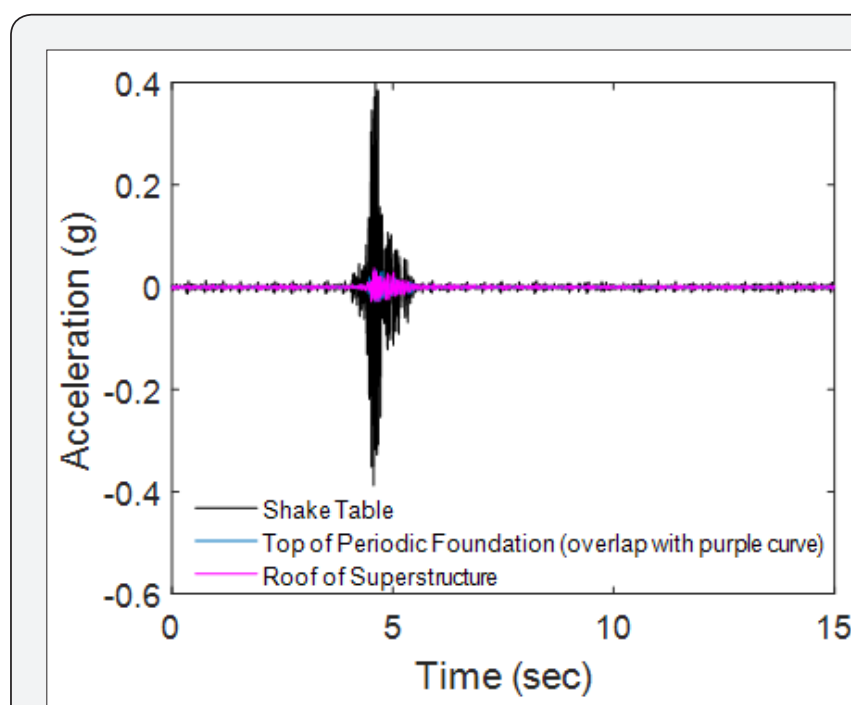

(a)

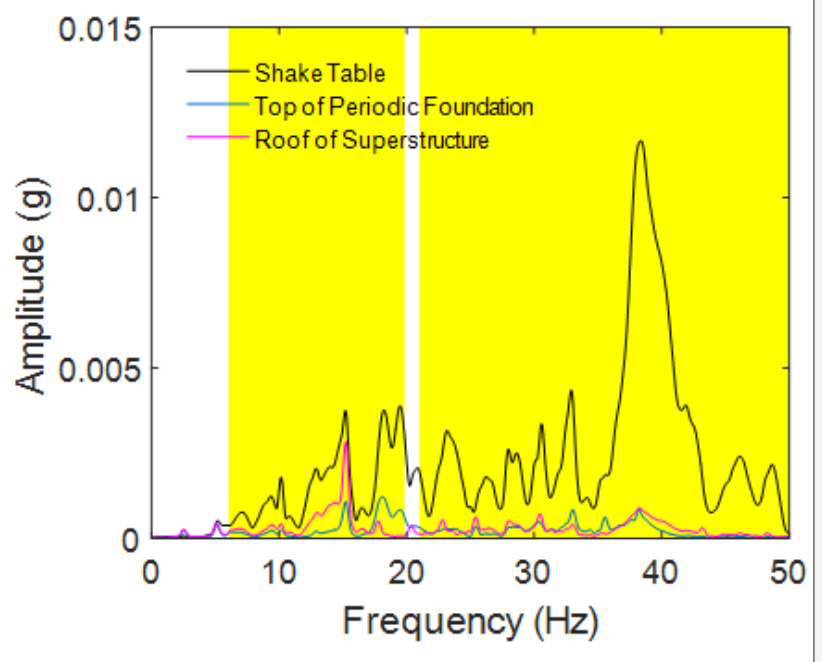

(b)

Figure 6 : Test results in (a) time domain; (b) frequency domain. 


\section{References}

1. Thomas EL, Groishnyy T, Maldovan M (2006) Phononics: Colloidal crystals go hypersonic. Nature materials 5(10): 773-774.

2. Xiang HJ, Shi ZF, Wang SJ, Mo YL (2012) Periodic Materials-based Vibration Attenuation in Layered Foundations: Experimental Validation. Smart Materials and Structures 21: 112003.
3. Yan YA, Laskar Z, Cheng F, Menq Y, Tang YL, et al. (2014) Seismic Isolation of Two Dimensional Periodic Foundations. Journal of Applied Physics 116: 044908-044901.

4. Yan Y, Cheng Z, Menq F, Mo YL, T, Shi Z (2015) Three Dimensional Periodic Foundations for Base Seismic Isolation. Smart Materials and Structures 24: 075006 . 